\title{
Késultats d'études théoriques et expérimentales concernant les phénomenès de polarisation provoquée dans le cadre d'applications géophysiques $\left({ }^{*}\right)$
}

\author{
1). TOURNIER
}

Riceived on February $91 \mathrm{~h}, 1972$

Sumsar. -.. The present treatise can be subdivided into four sections.

The first is concerned with I. P. phenomena in greneral, the diverse ways in which one may study such a phenomenon, the different parameters govern. ing it or permitting us to define it. A parallel is also drawn between the induced polarization and resistivity methods.

The second section is essentially theoretical. An effort is marle to sche. matize as much as possible a certain number of idealized typical cases for which the polarization produced by the passage of a continuous current may be calculated with precision, for two- or three-dimensional siructures. In these calculations we assume that the duration of passage of the current is sufficiently long for maximum polarization to be at tained. These hypotheses. necessarily and voluntarily oversimplified, have no other purpose than fo orient the person who is to interpret the phenomenon in question, to help him to comprehend the mechanisms brought into play therein -.- hence to guide him when ho is confronted with the much more complex results yielded by actual field surveys. One is thus obliged to recognize that despite a certain parallel between the techniques of classical electrical somnding and I. P. sounding, the analogy must by no means be drawn too closely. The overall aspect of the two types of sombling is not at all the same.

Although such calculations are highly useful, there is, none the less, a world of difference between highly simplified examples and antual problems. This gives rise to the necessity for an internediate stage for which only an experimental study by analogy, carried out on models, can be taken under consideration.

The third section is consequently experimental and deals precisely with morlels.

$\left(^{*}\right)$ These sontenue devant la Commission: L. Cagniard, President; J. Delloue, .J. Gognel, Examinateurs. 
Attention is first griven to the very simple case of a linear cell, that is, to a one-dimensional structure. A sturly is made of the interface potential and of its dependence upon the principal variables such as current density, time, etc. Thence we move on to the reneral problem of the analogical representation of the I.P. field by means of the "electric tank" A Alescription is given of such a tank - - its disposition, the materials as well as the measuring devices userl.

secomdly, different models corresponding as nearly as possible to the theoretical cases, previously calculated were thus able to be put to the test in the electric tank: horizontal plane, vertical half-plane, paralleliped, sphere, etc. Ilence it was possible to compare the experimental results with the theoretical calculations and to note a very satisfactory agreement hetween then. Ilaving recourse to models is therefore found to he justified whenever the actual structures become far too complex to become the object of calculations.

The fourth part describes the results of the various tests associated with the actual application of the process in the field.

Accordingly, independently of the results already published under the name of Roussel, one will find a rather spectacular study of the Soulier semi-conducting sulphides in the Gard (France). The hauxite deposits of the Var (France) have posed a more difficult problem. Another difficult problen which may be classified as a structural sturly was the search by indirect means of possible uranium-bearing deposits near the basement. $A$ study was likewise conducted in the Charite-sur-Loire (Nièvre, France) area.

As concerns deep probes, one may findit to his advantage to substitute the " lipole " electrode array for the classical symmetrical "Schlumberger" or "Wenner" array. A description of the particular study carried out by means of this former array appears in an appendix.

Rrassunto. - Il presente studio può essere sudliviso in quattro sezioni :

La prina riguarda i fenomeni di polarizazione indotta in generale, i vari modi in cui si possono studiare i fenomeni ed i vari parametri che li determinano o ci consentono di definirli. Viene tirata anche una parallela tra la polarizzazione indotta ed i metodi di resistenza.

La seconda sezione ì essenzialmente teorica. In essa si cerca di sehematizzare il più possibile un certo numero di casi tipici idealizzati nei quali la polarizzazione ottenuta dal passaggrio di una corrente continua può essere calcolata con precisione per strutture bi- o triclimensionali. Si presume in questi calcoli che il passagrio della corrente duri sufficientenente a lungo per raggiungere polarizzazioni massime. Queste ipotesi sono necessarianente e volutamente semplificate fino all'estremo. 11 loro unico scopo è di orientare chi deve interpretare il fenomeno, di aintarlo a capire il meccanismo applicato, cioè di gruidarlo quando si trova di fronte ai risultati molto più complessi ottenuti da veri rilevamenti. Si è così costretti a riconoscere che, malgrarlo una certa parallela tra le tecniche del classico sondagrio elettrico e del sondaggio a P. I., l'analogia non dev'essere con- 
siderata nel vero senso della parola. Iiaspetto complessivo dei due tipi di sondaggio ì tutt'altro che uguale.

Benchó tali calcoli siano utilissimi, esiste cionondimeno nna infinita di differenze tra gli esempi fortemente semplificali ed i veri problemi, per cui occorre creare una fase intermedia nella quale potri exsere preso in considerazione soltanto uno studio sperimentale per analogia. condotto su dei modelli.

La terza sezione, di conseguenza, è sperimentale e tratta precisamente di modelli. Viene esaminato prima il caso semplicissimo di una cellula lineare, cioè di una struttura unidinensionale. I)allo studio del potenziale interfacciale ed il modo in cui dipende dalle principali variabili quali densiti di corrente, tempo ecc., si è passati al problema generale della rappresentazione analogica della polarizzazione indotta a mezzo del "sesbatoio elettrico". Segue una descrizione di tale serbatoio, della sua disposizione, dei materiali usati e dei suoi sisteni di misura.

Successivamente si è cosi riusciti a provare, nel serbatoio elettrico. modelli vari corrispondenti il pin strettamente possibile ai casi teorici precerlentemente calcolati, ossia il piano orizzontale, il semipiano verticale, il parallelepipedo, la sfera ecc. E stato quindi possibile confrontare i risultati sperimentali con i calcoli teorici il che fece notare ma molto soddisfacente concordanza. Il ricorrere a modelli ̀̀, quindi, giustificato ogni volta che le strutture reali diventano troppo complesse per prestarsi a calcoli.

La quarta parte descrive i risultati delle varie prove in relazione alla vera applicazione del processo alla pratica. Di conseguenza, si troveri uno studio piuttosto nutrito sui solfuri semiconduttori di Soulier nel Gard (Francia), indipendentemente dai risultati gia pubblicati sotto il nome di Roussel. I giacimenti di bauxite del Var (Francia) rappresentarono un problema più arduo. Altro problema difficile -- che può essere classificato uno studio di struttura - era costituito dalla ricerca, con mezzi indiretti, di eventuali griacimenti di minerali di uranio vicino alla base. L'n altro studio è stato condotto a Charité sur Loire nel Dipartinento di Nièvre in Francia.

Per quanto riguarda i sondaggri in profondità, può essere vantaggioso sostituire la disposizione "dipolare" degli elettrodi alla classica disposizione simmetrica del tipo "Schlumberger" o "Wenner". Segue in ma appendice una descrizione dello studio particolare condotto secondo tale nuova disposizione. 
P'ages

AуаNт-PLUPOS . . . . . . . . . . . . . . . . . . . . 133

INTRODUCton . . . . . . . . . . . . . . . . . . . . 135

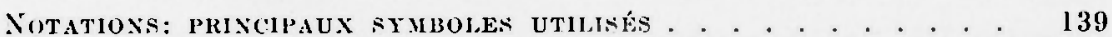

\section{PREMIERE PARTIE \\ CARACTERES PIIYSIQUES GENERAUX DU PIENOMENE DE POLARISATION PROVOQUEF:}

Cinapitre I: Phénomènes de Polarisation provoquée du sous-sol . .

1) Linéarité des phénomènes de polarisation dans le temps et en fonction du courant dexcitation. . . . . . . . . . . . . 146

2) Paramètres mesurés . . . . . . . . . . . . . . . . . . 147

3) Régulation de la source en technique transitoire . . . . . . 149

4) Différents nodles d'études transitoires . . . . . . . . . . 150

Cinapitre II: Comparaison entre les methodes des Résistivité ef de Polarisation provoquée . . . . . . . . . . . . . . .

1) Potentiel de polarisation d'un élément de volume dv dans un champ) électrique radial . . . . . . . . . . . . . . . . 152

2) Dispositif mono-électrodle (A, II) . . . . . . . . . . . . 155

3) Théorème de superposition et de réciprocité. . . . . . . . 156

4) Lois de similitudes. . . . . . . . . . . . . . . . . . . 157

5) Profondeur d’investigration . . . . . . . . . . . . 160

\section{DEUXIEIE PARTIE}

DISTRIBUTION GEOMETRIQUE DU CHAMP TILORIQUE DE POIARISATION PLOVOQUEE DE STRUCTURES SIMPLES

Chapitre 1: La Polarisation provoquée dans le cadre des phónomènes inclépendants du temps

1) Potentiel et champ à l'intérieur d'un volume polarisó

2) Potentiel et champ produit en un point extérieur par un volume polarisé . . . . . . . . . . . . . . . . 167

3) Influence du demi-espace isolant sur le potentiel de P.P. . 
Cuapror: II : Modeles mathematiques en champ uniforme.

1) Cylindre circulaire droit.

1.1 Potentiel dun cylindre droit polariso parallement in son axe. . . . . . . . . . . . . . . . . . . . .

1.2 Calcul de la composante horizontale du champ blectrique

1.3 Cas particulier du dispositif gradient noyen. . . . . . .

1.4 Construction des abaques . . . . . . . . . . . . . .

1.5 Ctilisation des abaques. . . . . . . . . . . . . 178

2) Siphìre polarisce . . . . . . . . . . . . . . . . 179

Cnaprtre: III: Modeles mathimatiques en champ radial . . . . . . 182

1) Potentiel de polarisation du filon mince vertical . . . . . . 182

2) Différence de potentiel de Polarisation provoquée d'un filon mince vertical indófini de hanteur variable . . . . . . . .

3) Potentiel de polarisation dun ruban horizontal indofini de lar-

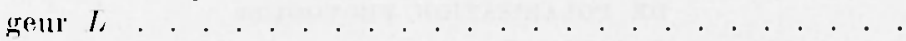

4) Potentiel de polarisation l'une conche mince horizontale indéfinie.

5) Calcul de la polarisabilité drune conche (quaisse indófinie. .

(5) Potentiel de polarisation du filon mince vertical infiniment risistant.

7) Potentiel de polarisation d'un filon mince vertical infiniment conclucteur.

Chaprtal: IV: Rófraction des lignes de courant. . . . . . . . 212

1) Polarisation volumique: filon rósistant . . . . . . . . . 213

2) Rófraction dans le cas d'un filon conducteur. . . . . . . 214

\section{TROISIENE PARTIE}

DISTRIBUTION DU CIAMP EXPEIMENTA DE POLARISATION PROVOQUEE

Cnapitis I: Etude expérimentale diun potentiel dinterface . . 219

I) Potentiel d'interface et surtension . . . . . . . . . . . 221

2) Type de cellule de mesure utilisée . . . . . . . . . . . . 222

3) Rósultats expórinentanx . . . . . . . . . . . . . . . . 224

3.1 Variation de $V_{\Delta}$ en fonction le $x$. . . . . . . . . . . 224

3.2 Mesure de $A v(A)$ (f.c.e.m. de polarisation) . . . . . . . 225

3.3 Variation de la f.c.e.m. de polarisation en fonction du temps 226

3.4 Variation de la f.c.e.m. de polarisation en fonction de $|i| 228$

3.5) Dópendance de la f.c.e.m. de polarisation vis in vis d'antres facteurs................. . . 228 
1'ages

1) Similitudes en Polarisation provoquée . . . . . . . . . . 231

1.1 Considérations génélales . . . . . . . . . . . . . 231

1.1.1 Problèmes de similitude en Polarisation provoquée 232

I.l.2 Conditions anx linites. . . . . . . . . . . . . 233

1.1.3 Conditions particulières . . . . . . . . . . . . 233

1.2 Appareillare de mesure . . . . . . . . . . . . . 233

1.2.1 Almentation en courant continu . . . . . . . . 234

1.2.2 Mesure de la d.d.p. et du champ électrique de polarisation. . . . . . . . . . . . . . 235

1.2.3 Programmateur . . . . . . . . . . . . . . . . 236

1.2.4 Nesure de la d.d.p. d'excitation . . . . . . . . . 236

1.3 Guves électriques et matériaux utilisés . . . . . . . . . 239

2) Quelques résultats expérimentanx . . . . . . . . . . . . 241

2.1 Conche mince indéfinie polarisce radialement . . . . . 243

2.1.1 Dispositif mono-électrode. . . . . . . . . . . . 243

2.1.2 Dispositif Wenner . . . . . . . . . . . . . . . 246

2.2 Volume parallèlépipèdique polarisé dans un champ uniforme 248

2.2.1 Observations préliminaires . . . . . . . . . . . 248

2.2.2 Etude du modèle polarisable . . . . . . . . . 251

2.3 Filons minces indéfinis polarisés dans un (hamp uniforme 257

2.4 Sphère polarisée dans un champ nuiforme . . . . . . . 258

\section{QUATRIEME PAR'IE}

\section{QUELQUes ExEMPLES DE PROSPECTION DE TERRAIN PAR LA METIIODE DE POLARISATION PROVOQUEE}

Cuapitre I: Appareillage de prospection "Polarisation provoquéen" 263

1) Caractéristiques du générateur de courant continu . . . . . 263

2) Ja voiture-laboratoire. . . . . . . . . . . . . . 264

Cuapitre II: Etude d'un gisement de sulfures conducteurs: le gisement pyriteux du Soulier . . . . . . . . . . . . . . . . . 266

I) Sondages $S W_{1}$ et $S W_{2} \ldots . . . . . . . . . . . .20260$

2) Profils $P_{1}$ A et $P_{2} \mathrm{IV}$. . . . . . . . . . . . . . . . . . 267

3) Interprétation quantitative des sondages P.P. . . . . . . . 269

Cuapitre III: Application à l'étude d'un gisement sódimentaire: les bauxites . . . . . . . . . . . . . . . . . 270)

1) Résultats des mesures de terrain et leur interprétation . . . 271

1.1 Structure du Nicollet. . . . . . . . . . . . 271

1.1.1 Coupe géologique . . . . . . . . . . . 271

1.1.2 Sondage Wenner $n^{\circ} 1 \ldots \ldots 271$

1.1.3 Profil $1^{\circ} 1$. . . . . . . . . . . . . . 274 
$1+1+$ Profil 11" 2 - $27+$

1.1.5 Profil 11" 3 . . . . . . . . . . . . . . 274

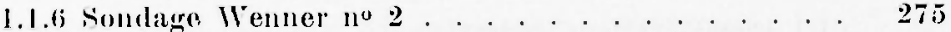

1.2 structure de la Baume Sud . . . . . . . . . . . . . 275

1.2.1 Plan de position . . . . . . . . . . . . . . 275

1.2 .2 Coupe grólogique $n^{\circ} \mathrm{I}$. . . . . . . . . . . . . 276

1.2 .3 Sondagre "deux ólectrodes" . . . . . . . . . . 277

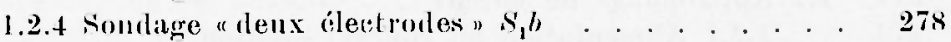

1.2 .5 Compe góologrique no 2 . . . . . . . . . . . . . 279

1.2.6 Sondage Wenner I centre en $S_{2}$. . . . . . . . 279

2) Interprótation quantitative de lanomalie de Lnnca sprie . . 280)

3) Conchusion . . . . . . . . . . . . . . . . . . . . . 281

Cunprtre IV: Recherches de minerais uranifores: problemes structurallx... . . . . . . . . . . . . . . . 282

1) Mesures de Polarisation provoqucie sur des echantillons de minerais dinaninm . . . . . . . . . . . . . . . . . . 282

2) Etude du bassin de Blanzy-Bert . . . . . . . . . . . . . 284

2.1 Sondage sur le socle cristallin . . . . . . . . . . . . . 285

2.2 Sondages sur le sódlimentaire . . . . . . . . . . . . 285

2.3 Profils P'. '. et résistivité . . . . . . . . . . . . . . 289

2.4 sondage profond de determination du socle . . . . . . 290

3) Conclusion . . . . . . . . . . . . . . . . . . . . . . 291

Cuapitre $V$ : Etude électrique dine structure localise par une ano. malie mannítique. . . . . . . . . . . . . . . . . . . . . 292

1) Mesures effectuces . . . . . . . . . . . . . . . . . . . 292

I.I fondage ilectrique Wenner . . . . . . . . . . . . . 292

1.2 Somblage deux ólectrode;. . . . . . . . . . . . . . . 294

1.3 Antres clispositifs . . . . . . . . . . . . . . . . . . 295

2) Conclusion . . . . . . . . . . . . . . . . . . . . . . . 297

\section{ANNLXE}

SONDAGE ELECTRIQUE PROFONII MISPOSITI MIPOLE . . . . . . . . 297

1) Remarques sur le dispositif dipôle. . . . . . . . . . . . 298

2) Sondage ílectrique équatorial hilatiral . . . . . . . . . . 301

3) Sondages dectriques Wenner et Schlumberger . . . . . . 302

('onclusion . . . . . . . . . . . . . . . 303

REFERENCE BIBLIOGRAPIQUES CITÉES . . . . . . . . . . . . . 305

BHBLIOGRAPIIE GÉNÉRALE UU SUJET . . . . . . . . . . . . 307

Liste des Figures . . . . . . . . . . . . . . . . . . . . . . 322 


\section{AVANT'PRUPUS}

Le travail qui fait l'objet de ce mómoire, s’il a quelque mérite. na até possible que grace it un ensemble de collaborations que je suis heureux de mettre en relief aujourd hui.

Toul dabord et on particulier, je tiens a exprimer ma plus profoncle reconnaissance et mon plus grand respect it Monsieur le P’rofesseur Cagniard, qui menseigna la géophysique appliquée et monedueillit dans son laboratoire. sa haute direction scientifique en môme temps que ses qualites humaines. nont cessi de me servir de guide ef de mencourager dans mon travail.

Jiensemble du Laboratoire de Góphysique Applique du Gentre de Recherches Géphysiques de Garchy, qui servit de carle i cette ríalisalion, cróa les conditions intellectuelles et maturielles necessaires a la bonne marche de celle-ci.

Je tiens tout d'ahoral à remercier particulièrement mon collègue et ami J. Roussel, actuellement Iaître de Conférences à Alger avec lequel la collaboration sur un même sujel permit les gchanges les plus fructeux. Son mómoire fut e.rit en Juin 1967.

Cest avec plaisir que jexprime igalement mes plus vifs remerciements a Monsieur J. J. Bureau, Maître-Assistan au Iaboratoire de Góophysique Appliquée, toujours prêt ì répondre aux plus clélicates questions mathematiques.

Le Bureau de Galcul du Laboratoire animo par M. Pham Van Ngoc secondé par mlle Boyer me rendit les services les plus compétents.

La ríalisation de lensemble de lappareillage de terrain fut menc ì bien par M. Beaussillon, Ingénienr, que je tiens à remercier ici particulièrement ainsi que toute son ípuipe, notamment M. Tort.

C'est avec plaisir que je remercie ici II. Duprez, notre Arministrateur ainsi que son pródécesseur M. Je Feuvre, qui facilitèrent lutilisation des divers services du laboratoire.

("est également l'ensemble du personnel et de l'Atelier de mécanique et plus particulièrement son Chef II. Lacourieux, que je tiens à remercier bien vivement pour toutes les róalisations menées a bien.

Le Bureau de Dessin et de Reproduction, particulièrement M. Banteaux et. II. Jalquin se chargèrent de la mise en forme du prisent manuscrit et Melles Lurier et Thomas assurèrent le travail clactylographique. 
Qu'il me soit permis de leur adresser à tous mes sincères remerciements, ainsi qu’à tous ceux qui à un titre ou à un autre, indirectement peut-être. participèrent à ce travail.

Parmi les organismes extérieurs qui ont permis ou facilité ce travail il $y$ a lieu de faire mention de la D.G.R.S.T. (1)élégation Générale à la Recherche scientifique et Technique), qui par la convention d'Action Concertée $n^{\circ} 63 \mathrm{~F} \cdot \mathrm{R}$ (080 donna l’impulsion financière nécessaire. Le B.R.G.H. (Bureau de Recherches (ióologriques et Minières) avec lequel nous avons collaborć, lors de cette Action Concertée et particuliomenent le Chef du Département Géophysique, H. Bollo, doivent être également remerciés pour leur aide. Les expóriences de terrain ont été rendues possibles grâce à l'accord bienveillant de diverses sociétés on organismes (Péchiney-Saint-Gobain, Péchiney, (.F.A. ...). Nous leur exprimons ici nos remerciements. 


\section{INTRODUCTION}

Voilà un peu plus d'un demi-siècle que Conrad schlumberger, à l'époque Professeur à l'école des Mines de Paris, imagina un principe nouveau de méthode de prospection électrique minière, méthode qu'il baptisa "Polarisation provoquée" (par abréviation P.P.). Une expérimentation assez poussée l'amena en 1920 à s'exprimer comme suit, dans son "Etude sur la prospection électrique du sous-sol "(GauthierVillars, Paris).

"Ce chapitre traite un sujet très différent de ceux exposés jusqu'ici. Il s'agit de différence de potentiel existant temporairement dans le sol à la suite du passage d'un courant électrique. Isa conductibilité plus ou moins grande des roches n'intervient plus dans les phénomènes étudiés qu'à titre accessoire.

" Ise but que je me suis proposé en abordant ces expériences itait de localiser les gisements à conductibilité métallique, par l'observation des phénomènes d'électrolyse que provoque à leur surface le passage dans le sol d'un courant de sens constant.

" Si l'on débite entre denx prises de terre $A$ et $B$ un courant $i$ de sens constant ... on provoque ainsi la polarisation du gisement c'està-dire que celui-ci se transforme en une véritable pile secondaire. Lorsque l'on coupe le courant électrolyseur $i$ cet accumulateur se décharge à travers le sol ... Ijobservation à la surface du sol des d.d.p. dues an courant de décharge doit permettre de localiser l'amas conducteur".

Schlumberger sut établir une distinction essentielle entre deux phénomènes en cause; d'une part la polarisation des électrodes d'émission, d'antre part la polarisation du volume global de terrain. Malgré les succès obtenus sur divers gisements, à Sain-Bel près de Lyon notamment, ce pionnier qui ne disposait pas à l'époque des moyens technologiques actuels, n'exploita pas plus avant la P.P. et préféra porter son effort sur les méthodes de résistivité, de sondage électrique, de carottage, de P.S. et même de courant tellurique.

C'es recherches anciennes semblent être alors tombées dans un oubli total jusqu'après la deuxième Guerre Mondiale. Désignée désormais 
"Polarisation induite" (Induced Polarization, par abréviation I.P.) la P.P. fait lobjet de publications américaines et canadiemnes (Bleil 1953, Seigel 1959, Frische et Von Buttlar 1957, Marshall et Madden, 1959). On doit a Wait (1959) une étude exhaustive à la fois theorique et expérimentale. Cette littérature mentionne des résultats spectaculaires obtenus sur des gîtes à minéralisation disséminée, de fatbles teneurs et des très gros tomnages.

lans le même temps, Vacquier (195i) revendique l'application des méthorles P.P. à la recherche des eanx douces souterraines.

Jes Russes de leur côté, n'étaient pas inactifs et l'on peut citer pour la seule année 1957, Kunlimov et Kotov, Komarov et Semenov, Rokityanski. Li Institut de Recherches et Techniques Geophysiques de Iséningrad (V.I.T.R.) semble avoir participé activement à cette invesligation. On peut citer également les Allemands Nosske (1959) at Buchheim (1957), l'I talien Belluigi (1956) et le Yougoslave Sumi (1959).

En France, le Professeur L. Gagniard proposa à J. Roussel, du Centre de Recherches Géophysiques (C.R.G.), d'aborder au laboratoire sur modèles réduits, l'étude physique systématique de ces phénomènes. Ln tel renouveau d'intérêt pour la P.P., les premiers résultats de Roussel, enfin la prise de conscience des Mineurs de la nécessité d'exploiter désormais de nouvelles ressources minérales provenant de gites a gros tonnages et faibles teneurs difficiles à mettre en évilence par les méthorles classiques, incitèrent en 1963 la Délégation Générale à la Recherche Scientifique et Technique (1).G.R.S.T.) à promouvoir la technique P.P. sur un plan industriel. C'est ainsi qu'une Action Concertée associa le Bureau de Recherches Géologiques et Minières (B.R.G.II.) et le Centre de Recherches Giophysiques du C.N.R.S. (C.R.G.).

Au C.R.G., les opérations furent conduites par J. Roussel, déja cité, et par D. Tournier, l'auteur du présent mémoire.

Cette Action Concertée s'engangeait cette fois sous les meilleurs auspices, comme suite des travaux préparatoires de Roussel, et en raison du fait que pouvait désormais être construit l'appareillage morlerne, puissant et sensible qui permettrait de mener à bien les expériences sur le terrain.

Au B.R.G.M. fut confice la variante d'application du procédé, fonlíe sur l'emploi des courants alternatifs de très basse fréquence (méthorle dite "fréquentielle»). Pour sa part, l'équipe Roussel-Tournier, du C.R.G., prit en charge les problèmes théoriques, expérimentanx et pratiques soulevés par la mise en oenvre du procédé, appliqué selon les vues initiales de Conrad schlumberger (méthode dite "transitoire"). Il s'agit 
d'injecter un courant continu, plus ou moins prolongé, pour polariser artificiellement le sous-sol. Après coupure du courant de charge, on étudiera le phénomène transitoire de dépolarisation.

Roussel ayant publié en 1967 , dans sa thèse, une description complète en détaillée de notre appareillage tel qu'il fut construit, mis au point et utilisé par l'équipe, il suffira ici de ne rappeler que très sommairement ses principes de construction et de mise en arurre.

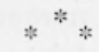

Le présent mémoire se subdivise en quatre parties.

La première traite des phénomènes de P.P. en général, des divers morles d'étude possible de ce phénomène, des difrérents paramètres dont. il dépend ou qui permettent de le caractériser. On établit aussi un parallèle entre la méthode de Polarisation provoquée et de Résistivité.

La deuxième partie est essentiellement théorique. On s'y efforce de schématiser au maximum un certain nombre de cas idéaux typiques pour lesquels la polarisation crécée par le passage d'un courant continu puisse être rigoureusement calculée, pour des structures ì deux ou à trois dimensions. Dans ce calcul on suppose que le passage du courant est assez prolongé pour qu'on atteigne la polarisation limite. Ces hypothèses nécessairement et volontairement simplistes n'ont d'autre objet que d'orienter l'interprétateur, de l'aider à bien pémétrer les mécanismes qui entrent en jeu dans le phénomène en question, par conséquent de le guider quand il se trouvera confronté avec les résultats beaucoup plus complexes de prospections réelles sur le terrain. On se trouve ainsi amené à constater qu'en dépit d'un certain parallèlisme dans les techniques respectives du sondage électrique classique et du sondage P.P., il ne faut absolument pas pousser l'analogie trop loin. I'allure générale des deux types de sondages n'est pas du tout la même.

Malgré l'intérêt de tels calculs, il subsiste néanmoins un abîme entre les schémas simplistes et les problèmes réels. Il en résulte la nécessité d'une étape intermédiaire, pour laquelle il n'est possible d'envisager qu'une étude expérimentale analogique, effectuce sur modèles résluits.

Ia troisième partie est done expérimentale et concerne précisément les modèles réduits. 
On y aborde en premier lien le cas particulièrement simple d'une "cellule lináaire ", c'est-à-rlire d'une structure à une seule climension. On éturlic le "potentiel d'interface ", sa rlépendance vis-à-vis des principales variables, telles que la densité de courant, le temps, etc. ... On passe alors au problème général de la représentation analogique du champ de P.P. par le moyen de la "cuve électrique ". On décrit cette ('uve, son agencement, les matériaux utilisés ainsi que l'appareillage de mesure.

En deuxième lieu, différents morlèles se rapprochant aussi exactement que possible des schémas théoriques précédemment calculés ont pu etre ainsi testés dans la cuve ilectrique: plan horizontal, demi-plan vertical, parallèlépipierle, sphère, etc. ... Il a rlonc été possible rle comparer les résultats experimentaux aux calculs théoriques et rle constater leur très satisfaisante concorrance. Ainsi se trouve justifié le recours aux morlèles rérluits, chaque fois que les structures réelles deviennent beaucoup trop complexes pour pouvoir être soumises au calcul.

La quatrième partie expose les résultats des tests variés auxquels a rlonné lien l'application concrète rlu procédé sur le terrain.

("est ainsi qu'indépendamment des résultats déjà publiés sous le nom rle Roussel, on trouvera une iturle assez spertaculaire des sulfures semi-conducteurs du Soulier dans le Gard. Les gîtes rle bauxite du Var ont posé un problème plus difficile. Un autre problème difficile se situant dans le carle d'une itude structurale, fut la recherche, par voie indirecte, de gîtes uranifères éventuels an voisinage du socle. Une éturle fut faite également dans la région de La Charité-sur-Loire (Nièvre).

Quand il s'agit d'investigations profondes, on peut trouver intírêt à substituer la disposition "dipôle-dipôle " à la disposition classiques symétrique "Schlumberger" ou "Wenner". L'éturle particulière faite au moyen re ce rlispositif est reportée en annexe. 


\section{NOTATIONS - PRINCILALX SYMBOLES UTILISES}

$$
a
$$

1

$A, B, a, b, K, K_{1}$ 13

d.1.p.

d. II

$d s$

d I'

$d v_{0}, d v_{x}, d v_{A}, d v_{H}$
$D=d / h_{1}$.

rayon le la sphère; distance $A B / 3$.

sources ponctuelles de polarisation de lélément de volume dis.

élertrode source ponctinelle.

('onstantes.

éleciroule puits ponctuelle.

diffirence de potentiol.

monent dipolaire de polarisation de lélóment de volume $d v$, de l'ólément de surface $d s$.

élóment rle surface.

intensiti dn courant traversant -a et - $\rightarrow$.

potentiel ólémentaire de polarisaion de l'ólóment ds en $o, M$, etc. ...

I) dianètre des faces du rylindre, exprinú en nnité de profondeur; déviation des lignes decourant ( $\left.\varphi_{1}-q_{0}\right)$.

$E, E_{n}, E_{y}, E_{x}, E_{z}, E_{l}, E_{n}$ vecteur rhamp dectrique et ses composantes. $\overline{E_{1}}$ champ électrique dû à la face 1 du cylindre: $0_{1}$ angrle $\left(E_{1}^{\prime}, \bar{\imath}\right)$.

$E_{2}$ champ électrique dû à la face 2 du cylindre: $\theta_{2}$ angrle $\left(\bar{E}_{2}, \bar{i}\right)$.

$E=e / h_{1}$ ipaisseur de la couche horizontale exprimée en nuité de profonileur.

$|E(0, k)|_{0}^{\pi / 2}$ integrale elliptique complète du seromd ordre.

f.e.m1.

$|F(0, k)| \begin{aligned} & \pi / 2 \\ & 0\end{aligned}$

$h_{1}$

$H=h / h_{1}$

$\bar{i}, i_{x}, i_{r}, i_{t}, i_{n}$

$\bar{i}, \bar{j}, l$

I

$I, I_{1}, I_{2}$ force slectromotrice.

intégrale elliptique complète de logendre du premier orilre.

profondeur mito des diffórents morlèles throriques ot expérimentaux ot de lélément de volume $d v$.

hauteur riduite du filon vertical.

verteur densité des conrant électrique.

vecteurs unitaires des axes, o.x, oy, oz.

intensité du courant d'excitation, constante, traversant la source $A$ ou $B$, en ampères génciralenent.

valeurs des integrales diverses. 
$l, l^{\prime}$

$i_{1}$

$\mathrm{li}_{2}$

$l=A B / 2$ et $l=. \mathrm{H} / 2$

$l=l h_{1}$

$\bar{W}$ et $W^{\prime}$

.$V$ ef $x$

$"$

$\bar{p}$

I'.I'.

1 ,

$\bar{r}$

$r, r^{\prime}, r^{\prime \prime}, r_{x}, r_{N}$

(li. \%, Z)

1

10

polarisabilité de l'élément de volume dr ou des volumes $r$ ef $r$.

rapport de similitude géometrique.

rapport de similitude des polarisabilités.

largour du ruban horizontal exprimée en unité de profondeur; hauteur rédnite du eylindre droit: larrreur du filon.

moments de polarisation des volumes $r$ ef $r$.

ilertrorle: ponctuelles de mesure.

normale milaire à une surface.

moment dipolaire electrique.

Polarisation provoquée.

vecteur de polarisation álectrique.

verteur joignant deux points de coordomués (s, $1, h)$ At $(r, \%, z)$.

modules des rayons vecteurs correspondants.

coordommes cylimdriques.

temps variant de 0 is linfini.

instant initial douverture du circuit $A B$ pris comme origine des temps.

$r_{M} \cdot r_{x}, r_{A}, r_{A} \cdot r(A, M) \ldots$ polentiel soalaire de polarisation aux points $u, N, A, A,(A, M)$ etc. $\ldots$

ro tension transitoire a l'instant $1-0$.

$r .1(1)$

$r(l)$

tension transitoire: róponse is un créneau de durée 1 ì l'instant $1(\mathrm{en} \mathrm{mV})$.

tension transitoire: róponse à un échelon $(.1=\infty)$ à l'instant 1 en m $Y$ généralenent.

$V_{A}, V_{A}, V_{M}$

$r 1$

potentiel scalaire d'excitation aux points $A, A^{\prime}, .$. différence de potentiel variable suivant l'ótablisse. ment du comrant d'excitation $I$ (en volts).

$\Gamma_{\infty}$

limite de $V_{A}$ provoqué par le courant dexcitation constant $I$, quand $A$ tend vers l'infini (en volts).

$r$ et $r^{\prime}$

volumes homolognes polarises.

l $(x: y z)$

$(x, y, z)$

vecteur vitesse des charges b́lectriques.

coordonnes cartésiemes rectangulaires.

durée du crónean dexcitation en secondes on minutes. $1 r_{M N}, 1 r_{S}, 1 r_{w}$ rifférence de potentiel de polarisation entre $M$ et $N$, en dispositif schlumberger. Wenner. 
$\varepsilon$

0

$(\theta, R)$

(1), $\varphi, r)$

$(\xi, \eta, h)\left(\xi^{\prime}, \eta^{\prime}, h^{\prime}\right)$

o, $\underline{g a}$

$\varrho=R / h_{1}$

$o^{\prime}$

$\sigma=1 / Q$

$\sigma^{\prime}$

$\varphi_{1}, \varphi_{2}$

$\%-x / h_{1}$

$\Omega_{1}$. SL.,

vecteur quelconque.

angle oriente entre le moment du dipôle $\bar{\rho}$ et la direc. tion du rayon vecteur $\bar{r}$.

coordomnces polaires à deux variables.

coorlonnées spliériques.

coordomuces dles points.$M$ et $M$.

résistivité vraie et apparente en courant continu (en olım.mètre).

coorlonnces róduites.

densite volumique de cluarge dectrique fictive.

conductivité électricue en courant continu.

densitó superficielle de charcre clectrique fictive.

angles faits par les lignes de courant avec la normale i. l"interface dans les deux milieux 1 at 2 .

abscisses réduites en unité de profondeur.

aucres solides.

\section{PARAMETRES MESURES}

$m_{a}(l)=v(t) / V_{\infty} \quad$ polarisabilite apparente i linstant $t$ secondes en $\mathrm{m} \mathrm{V} / \mathrm{V}$ en millièmes $\left({ }^{0} / 00\right)$.

III (t) polarisabilite vare d'un milieu lonomorène à l'instant $t$.

$a=\frac{m_{a}, t_{1}}{m_{a}, t_{2}}=\frac{v\left(t_{1}\right)}{v\left(t_{2}\right)}$ taux de dácroissance de la tension transitoire (saus (limension).

$\frac{1}{V_{\infty}} \int_{t_{1}}^{t .} v(t) d t \quad$ valeur le lintégrale exprimóe en mV/V secondes ou millisecondes.
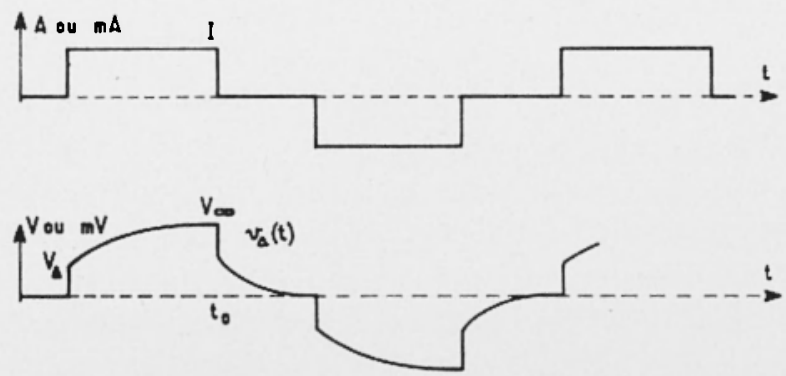